\title{
Resiliencia de la cobertura vegetal en el Suroeste de México ante los efectos del cambio climático
}

\author{
Resilience of vegetation cover in Southwest Mexico to the climate change effects
}

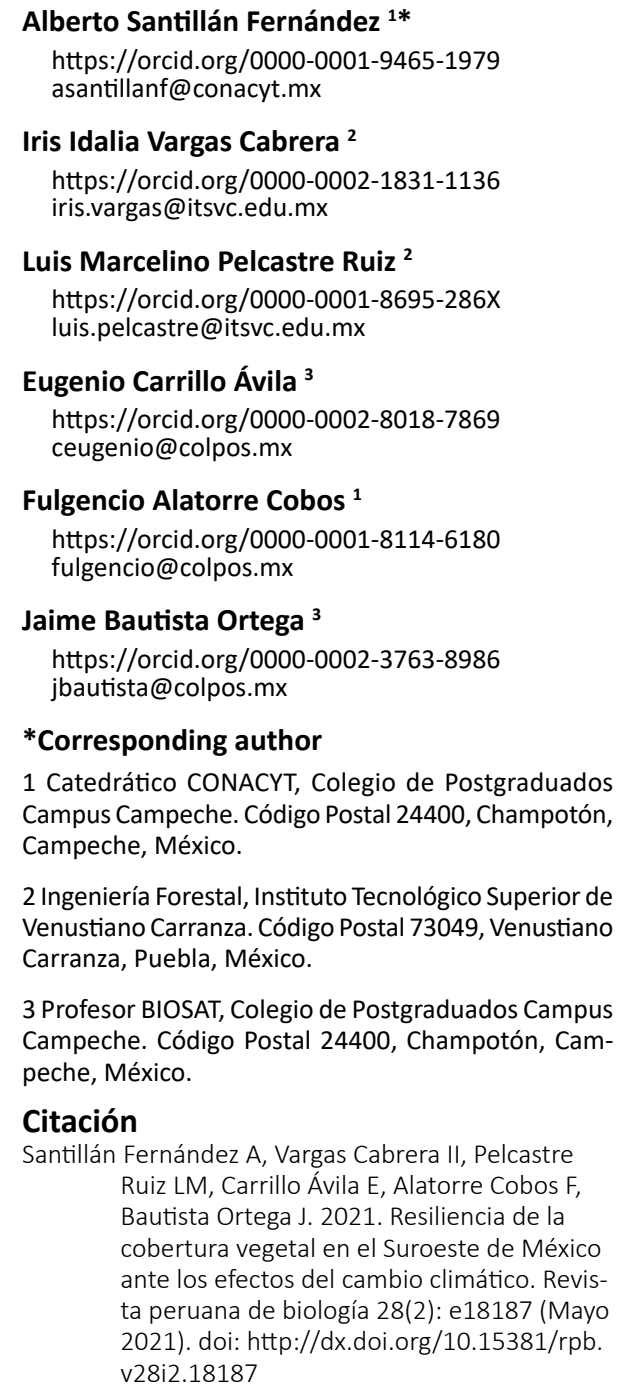

Presentado: $\quad 13 / 07 / 2020$

Aceptado: $\quad 27 / 02 / 2021$

Publicado online: 25/05/2021

Editor: Leonardo Romero

\section{Resumen}

El modelado de escenarios de cambios climáticos utilizando sistemas de información geográfica para estimar la resiliencia de la cobertura vegetal es una herramienta útil para proyectar impactos futuros e implementar estrategias de conservación o manejo. En el presente trabajo asociamos espacialmente la biodiversidad de la cobertura vegetal del Suroeste de México con su capacidad para adaptarse a los efectos del cambio climático. Para analizar esta asociación se estimaron índices de riqueza y diversidad de especies, y su relación con escenarios de clima futuro. Se utilizaron los registros geográficos del Inventario Nacional Forestal y de Suelos para ocho comunidades vegetales (arbórea, arbustiva, herbácea, palma, cactus, bejucos, helechos y xerófita) distribuidas entre Guerrero, Oaxaca y Chiapas. La proyección climática fue al 2050, con modelos de circulación global A2 (promedio CCCMA, HADCM3 y CSIRO), 19 variables bioclimáticas y una resolución de 2.5 minutos. Los escenarios de cambio climático se modelaron con el algoritmo MaxEnt y la riqueza de especies, índice de diversidad y regresiones espaciales con el software Diva-GIS v7.5. Los modelos de regresión espacial estimaron que a mayor riqueza y diversidad de especies mayor seria la resiliencia que mostraría el ecosistema. Las comunidades vegetales cactus, palma y xerófita mostraron mayor vulnerabilidad al cambio climático. Las variaciones en la estacionalidad de la temperatura resultó ser el factor que condicionaría su distribución futura. Por lo que, las estrategias de conservación o manejo deberían considerar a la diversidad como un agente del ecosistema que amortiguaría a los efectos negativos del clima futuro.

\section{Abstract}

The scenarios modeling of climate changes using geographic information systems to estimate the vegetation cover resilience is a useful tool to project future impacts and implement conservation or management strategies. We associate spatially the biodiversity of the vegetation cover of Southwest Mexico with its ability to adapt to the effects of climate change. We analysis this association estimating species richness and diversity indices, and its relationship with scenarios of future climate. Geographical records of the National Forest and Soil Inventory were obtained for eight plant communities (arboreal, shrubby, herbaceous, palm, cactus, vines, ferns, and xerophyte) distributed in Guerrero, Oaxaca, and Chiapas. The climatic projection was to 2050, with global circulation A2 models (CCCMA, HADCM3 and CSIRO average), 19 bioclimatic variables and a resolution of 2.5 minutes. Climate change scenarios were modelled with the MaxEnt algorithm and species richness, diversity index, and spatial regressions with Diva-GIS v7.5 software. The spatial regression models estimated that higher richness and species diversity, the greater resilience that the ecosystem would show. The cactus, palm, and xerophytic plant communities presented greater vulnerability to climate change. Variations in temperature seasonality turned out to be the factor that would condition its future distribution. Therefore, in conservation or management strategies, diversity should be considered as an agent of the ecosystem that cushions the negative effects of future climate.

Palabras clave:

Diversidad de especies; Ecosistema; MaxEnt; Riqueza de especies; Sistemas de Información Geográfica; Cambio Climático.

Keywords:

Species diversity; Ecosystem; MaxEnt; Species richness; Geographic Information Systems; Climate Change. 


\section{Introducción}

México se ubica en una zona de transición entre dos grandes regiones biogeográficas, la neotropical (Sudamérica y Centroamérica) y la neártica (Norteamérica), lo que le ha permitido desarrollar condiciones ambientales únicas con una gran variedad de hábitats y formas de vida (Halffter 2017). México es un país megadiverso, que alberga el $10 \%$ de la biodiversidad del planeta (Sarukhán et al. 2015). El sur de México es la región con mayor diversidad del país, donde habita el $70 \%$ de su diversidad biológica; destacando en orden de importancia, los estados de Oaxaca (con el mayor número de especies endémicas), Chiapas, Veracruz y Guerrero (Sarukhán et al. 2015). Sin embargo, por su situación geográfica, condiciones climáticas, orográficas e hidrológicas, el sur de México se encuentra en una de las zonas más vulnerables al cambio climático (Fuentes-Franco et al. 2015).

El cambio climático es la mayor amenaza que enfrenta la vida, porque eleva la temperatura promedio del planeta, afecta el ciclo del agua, la frecuencia de los fenómenos climatológicos normales y acentúa los desastres naturales, que conlleva a daños en comunidades, cultivos y ecosistemas, rompiendo el equilibrio ecológico en el cual se sustenta la vida actual en la Tierra (Brönnimann 2015, Whitney et al. 2017). El concepto de cambio climático paso de ser un tema de análisis para el futuro a un tema de investigación para el presente (Dumitrescu et al. 2015). Sus causas y efectos están altamente documentados e inclusive las predicciones mediante modelos geoestadísticos en el mediano y largo plazo son poco alentadoras para las formas de vida que se conciben actualmente (IPCC 2013).

El empleo de modelos de Sistemas de Información Geográfica (SIG), para predecir los efectos del clima futuro sobre la cobertura vegetal, es cada vez más frecuente por la simplicidad de su uso y la confiabilidad en sus resultados (Pliscoff \& Fuentes-Castillo 2011), lo que permite tomar decisiones en cuanto a la conservación o aprovechamiento de las especies, al determinar las variables climáticas que más inciden en su desarrollo (Phillips et al. 2006). Entre los SIG que modelan el nicho ecológico de especies vegetales a partir de variables climáticas en condiciones actuales y futuras, el algoritmo de Máxima Entropía (MaxEnt) tiene las mejores evaluaciones (Elith et al. 2011). Se ha aplicado con éxito en la ubicación de áreas vulnerables al cambio climático, lo que permitió tomar estrategias de conservación o manejo, en especies como Vasconcellea spp (Scheldeman et al. 2007), Pinus patula Schiede ex Schltdl. \& Cham. y Pinus tecunumanii F. Schwerdtf. (Van Zonneveld et al. 2009a), Swietenia macrophylla King (Garza-López et al. 2016) y Lysiloma latisiliquum (L.) Benth. (Garza-López et al. 2018).

En diversos lugares se han realizado estudios que predicen los efectos del cambio climático en la cobertura y diversidad de especies como los realizados en España (Lloret 2012), Europa (Lindner et al. 2014), sureste de Asia (Van Zonneveld et al. 2009b), norte de América (McKenney et al. 2007) y México (Van Zonneveld et al. 2009a, Monterroso-Rivas et al. 2013). Sin embargo, para México existe poca investigación que analice la diversidad de es- pecies como un factor de resiliencia y adaptación ante los efectos del clima futuro (Cuevas-Coeto et al. 2019).

La resiliencia es la capacidad que tienen los ecosistemas de absorber, amortiguar y resistir los cambios abióticos y bióticos (Moritz \& Agudo 2013, Santiago-Vera et al. 2018). Esta capacidad de recuperación o amortiguamiento es determinada por variables específicas asociadas con la regeneración como la composición de plantas, productividad, biomasa, acumulación de nutrientes en el suelo y diversidad ecológica (Chambers et al. 2019). Para estimar la resiliencia de especies en un ecosistema, el índice de diversidad de Shannon es un buen método (Dymond et al. 2014).

El IPCC (2013) estimó que una variación mínima en el clima futuro puede causar la extinción del $18 \%$ de las especies conocidas, mientras que un cambio máximo, provocaría la extinción del $35 \%$ de las especies. Al respecto Sakschewski et al. (2016) consideran que la resiliencia en comunidades vegetales es mayor que en grupos taxonómicos específicos, al ser una propiedad ecosistémica que deriva de la biodiversidad; por lo que los ecosistemas con mayor diversidad de especies presentan mayor probabilidad de adaptarse al cambio climático.

Bajo este contexto el objetivo del presente estudio fue asociar espacialmente la biodiversidad de la cobertura vegetal del Suroeste de México con su capacidad para adaptarse a los efectos del cambio climático, mediante la estimación de índices de riqueza y diversidad de especies, y su relación con escenarios de clima futuro obtenidos por sistemas de información geográfica.

\section{Materiales y métodos}

Área de estudio. En este estudio se consideraron los datos georreferenciados de distribución puntual de las especies vegetales del Suroeste de México, que incluyeron a los estados de Guerrero, Oaxaca y Chiapas (Figura 1), reportados por el Inventario Nacional Forestal y de Suelos (INFyS) (CONAFOR, 2014). Se homogeneizaron los nombres científicos y se omitieron aquellos registros con leyenda: sin identificar, en identificación o no definida. Cada especie se clasifico en alguna de las formas biológicas: árbol, arbusto, palma, cactus, hierba, helechos, xerófita y bejuco, conforme la clasificación propuesta por CONAFOR (2018).

Relación de la riqueza y diversidad de especies con los efectos del cambio climático. Para ubicar las zonas en el Suroeste de México donde coexiste el mayor número de especies de las diferentes formas biológicas, se delimitó espacialmente a la distribución georeferenciada de las especies, el índice de riqueza de especies (IRE) y el índice de diversidad de Shannon (IDS). Para ello se recurrió al software Diva-Gis v7.5 (Diva-GIS 2019) de herramientas SIG; las instrucciones fueron Species richness analysis para IRE y Species diversity analysis para IDS, conforme la metodología de Hijmans et al. (2001). Para ambos índices la resolución empleada fue de $2.5 \mathrm{mi}$ nutos lo que equivale a $5 \mathrm{~km}^{2}$. De acuerdo con Pliscoff \& Fuentes-Castillo (2011) la resolución de 2.5 minutos es una buena escala para estudios regionales. 


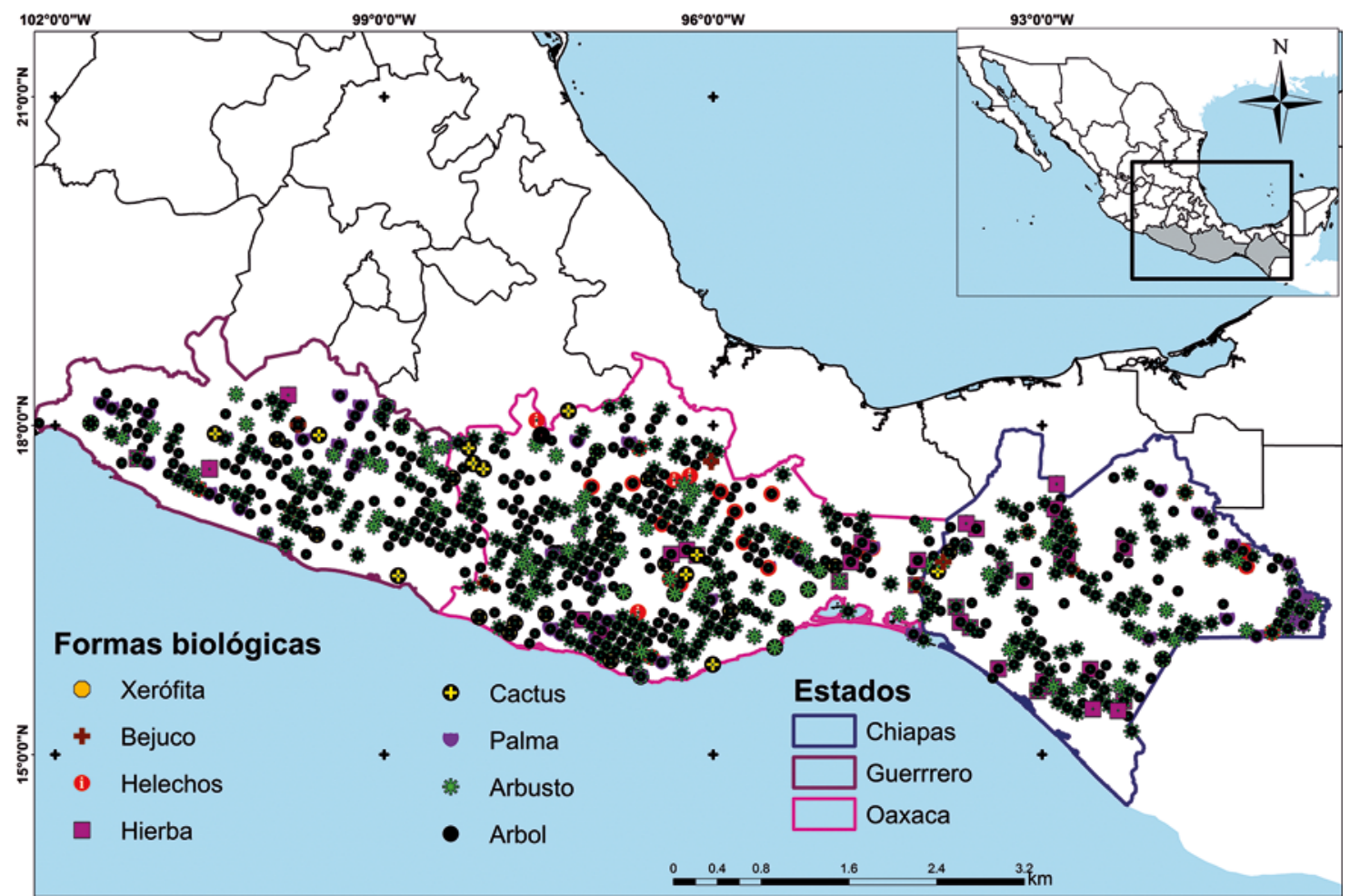

Figura 1. Ubicación espacial de las formas biológicas existentes en el área de estudio conforme los datos reportados por el Inventario Nacional Forestal y de Suelos.

Para asociar espacialmente la resiliencia que las áreas con mayor Riqueza (IRE) y Diversidad (IDS) de especies presentaron ante las variaciones del clima futuro, se estimó un modelo de cambio climático a la distribución puntual de las especies, conforme la metodología de Hijmans et al. (2005). La proyección climática empleada fue a 2050 con modelos de circulación global (promedio CCCMA, HADCM3 y CSIRO) bajo el escenario de emisión A2 que incluyó 19 variables bioclimáticas (IPCC 2013).

El análisis de los modelos de cambio climático (MCC) permitió generar 4 escenarios: (a) Áreas de bajo impacto: áreas donde una especie ocurre potencialmente en la actualidad y en el futuro (matemáticamente el modelo asigna valores de 1 a los pixeles resultantes), (b) Áreas de alto impacto: áreas donde una especie ocurre potencialmente en la actualidad pero para el futuro ya no es apta (pixeles con valores de -1), (c) Nuevas áreas aptas: áreas donde una especie ocurre potencialmente en el futuro, pero que en la actualidad no es apta (pixeles con valores de 2), y (d) Área no apta, ni bajo las condiciones actuales ni futuras (pixeles con valores de 0) (Hijmans et al. 2005).

Los puntos georreferenciados por formación vegetal, se introdujeron en el algoritmo de Máxima entropía (MaxEnt 2019) en formato delimitado por comas (.csv) y las variables climáticas en formato ASCII (.asc) con una resolución de 2.5 minutos. Los resultados se importaron a DivaGis v7.5 (DivaGis 2019) y se convirtieron en formato grig (.grd) para visualizarse como imágenes.

Para probar estadísticamente la bondad de ajuste de la resiliencia que las comunidades vegetales presentan ante los efectos del cambio climático, se contabilizaron la can- tidad de pixeles que coincidieron por cada uno de los escenarios climáticos $(-1,0,1$ y 2) con los valores obtenidos en el Î́ndice de Diversidad de Shannon, para ello se empleó la técnica del vecino más próximo de herramientas de SIG (González de Andrés et al. 2014). Además, conforme la metodología de Hijmans et al. (2005), se estimaron modelos de regresión espacial simple y múltiple en DivaGis v7.5 que consideraron los escenarios del MCC como variable dependiente y como independientes IDS e IRE.

Escenarios de cambio climático por tipo de comunidad vegetal. Para asociar la resiliencia que la diversidad de especies vegetales presenta en el Suroeste de México, se estimaron espacialmente MCC para cada una de las ocho comunidades vegetales presentes en el área de estudio y se comparó con el MCC que considera la suma de todas ellas (Biodiversidad). Además, se calcularon las coberturas en $\mathrm{km}^{2}$ por cada escenario del MCC y comunidad vegetal, así como la confiabilidad de las predicciones (AUC) y las variables climáticas que más contribuyen en dichas predicciones.

\section{Resultados y discusión}

En el Suroeste de México que comprende los estados de Guerreo, Oaxaca y Chiapas, la CONAFOR (2014) reportó 44272 sitios de muestreo, en los cuales se localizaron 730957 individuos pertenecientes a 8 comunidades vegetales (formas biológicas) (Tabla 1). En total se analizaron 164 familias, 454 géneros y 808 especies. Las comunidades arbóreas y arbustivas fueron las que presentaron mayor diversidad de familias, géneros y especies, sobre todo en los estados de Oaxaca y Chiapas; este aspecto ha sido ampliamente documentado por Sarukhán 
et al. (2015) y Halffter (2017) quienes estiman que en el sur de México se encuentra el 70\% de la diversidad nacional, atribuible a las condiciones edafoclimaticas que han permitido el desarrollo de los ecosistemas selva y bosque.
El estrato arbóreo presentó el mayor número de individuos con el $90.343 \%$, seguido del arbustivo (6.308\%), palma $(1.403 \%)$, cactus $(1.062 \%)$ y en menor grado herbáceas $(0.405 \%)$, bejuco $(0.243 \%)$, helechos $(0.123 \%)$ y xerófita $(0.114 \%)$ (Tabla 2$)$.

Tabla 1. Número de familias, géneros y especies de las comunidades vegetales en el Suroeste de México por entidad federativa.

\begin{tabular}{|c|c|c|c|c|c|c|c|c|c|c|c|}
\hline \multirow{2}{*}{$\begin{array}{l}\text { Forma } \\
\text { Biológica }\end{array}$} & \multicolumn{2}{|c|}{ Datos INFyS } & \multicolumn{3}{|c|}{ Guerrero } & \multicolumn{3}{|c|}{ Oaxaca } & \multicolumn{3}{|c|}{ Chiapas } \\
\hline & Sitios & Individuos & Familia & Género & Especie & Familia & Género & Especie & Familia & Género & Especie \\
\hline Arbol & 40012 & 660366 & 64 & 148 & 240 & 81 & 234 & 373 & 78 & 209 & 338 \\
\hline Arbusto & 2835 & 46111 & 20 & 32 & 38 & 30 & 57 & 91 & 33 & 63 & 87 \\
\hline Palma & 569 & 10252 & 1 & 5 & 5 & 1 & 7 & 8 & 1 & 8 & 8 \\
\hline Cactus & 433 & 7764 & 1 & 4 & 6 & 1 & 9 & 18 & 1 & 3 & 3 \\
\hline Hierba & 186 & 2961 & 2 & 3 & 3 & 5 & 6 & 6 & 11 & 13 & 19 \\
\hline Helechos & 81 & 898 & 2 & 2 & 2 & 3 & 3 & 11 & 2 & 2 & 3 \\
\hline Xerófita & 79 & 832 & 0 & 0 & 0 & 5 & 5 & 5 & 0 & 0 & 0 \\
\hline Bejuco & 77 & 1773 & 2 & 2 & 2 & 3 & 3 & 3 & 4 & 4 & 4 \\
\hline
\end{tabular}

Tabla 2. Géneros con el mayor número de individuos por comunidad vegetal que coexisten en el Suroeste de México.

\begin{tabular}{|c|c|c|c|c|c|c|c|c|c|c|c|}
\hline \multicolumn{3}{|c|}{ Arbol } & \multicolumn{3}{|c|}{ Helechos } & \multicolumn{3}{|c|}{ Arbusto } & \multicolumn{3}{|c|}{ Bejuco } \\
\hline Género & Núm. & $\%$ & Género & Núm. & $\%$ & Género & Núm. & $\%$ & Género & Núm. & $\%$ \\
\hline Quercus & 199250 & 27.259 & Alsophila & 460 & 0.063 & Montanoa & 3123 & 0.427 & Acacia & 698 & 0.095 \\
\hline Pinus & 59405 & 8.127 & Cyathea & 282 & 0.039 & Miconia & 2568 & 0.351 & Smilax & 538 & 0.074 \\
\hline Bursera & 23204 & 3.174 & Sphaeropteris & 156 & 0.021 & Plumeria & 2566 & 0.351 & Serjania & 196 & 0.027 \\
\hline Heliocarpus & 15253 & 2.087 & & & & Eysenhardtia & 2564 & 0.351 & Macfadyena & 97 & 0.013 \\
\hline Acacia & 13894 & 1.901 & & & & Morella & 2257 & 0.309 & Psychotria & 85 & 0.012 \\
\hline Clethra & 13831 & 1.892 & & & & Cnidoscolus & 2194 & 0.300 & Strychnos & 75 & 0.010 \\
\hline Arbutus & 13295 & 1.819 & & & & Croton & 2131 & 0.292 & Rourea & 54 & 0.007 \\
\hline Lysiloma & 12222 & 1.672 & & & & Randia & 2038 & 0.279 & Doyerea & 15 & 0.002 \\
\hline Alnus & 11838 & 1.620 & & & & Psychotria & 1693 & 0.232 & Solanum & 15 & 0.002 \\
\hline Cordia & 8871 & 1.214 & & & & Senna & 1418 & 0.194 & & & \\
\hline Otras (293) & 289303 & 39.579 & Otras (0) & 0 & 0.000 & Otras (84) & 23559 & 3.223 & Otras (0) & 0 & 0.000 \\
\hline Total (303) & 660366 & 90.343 & Total (3) & 898 & 0.123 & Total (94) & 46111 & 6.308 & Total (9) & 1773 & 0.243 \\
\hline \multicolumn{3}{|c|}{ Cactus } & \multicolumn{3}{|c|}{ Hierba } & \multicolumn{3}{|c|}{ Palma } & \multicolumn{3}{|c|}{ Xerófita } \\
\hline Género & Núm. & $\%$ & Genero & Núm. & $\%$ & Genero & Núm. & $\%$ & Genero & Núm. & $\%$ \\
\hline Stenocereus & 2556 & 0.350 & Verbesina & 1585 & 0.217 & Brahea & 4878 & 0.667 & Nolina & 324 & 0.044 \\
\hline Pachycereus & 1405 & 0.192 & Gomphrena & 312 & 0.043 & Cryosophila & 3704 & 0.507 & Agave & 203 & 0.028 \\
\hline Pilosocereus & 1182 & 0.162 & Ayenia & 236 & 0.032 & Sabal & 1127 & 0.154 & Beaucarnea & 154 & 0.021 \\
\hline Neobuxbaumia & 633 & 0.087 & Peperomia & 127 & 0.017 & Acrocomia & 158 & 0.022 & Yucca & 116 & 0.016 \\
\hline Opuntia & 628 & 0.086 & Hyptis & 123 & 0.017 & Bactris & 114 & 0.016 & Dasylirion & 35 & 0.005 \\
\hline Escontria & 421 & 0.058 & Cuphea & 104 & 0.014 & Astrocaryum & 77 & 0.011 & & & \\
\hline Myrtillocactus & 399 & 0.055 & Euphorbia & 64 & 0.009 & Scheelea & 70 & 0.010 & & & \\
\hline Cephalocereus & 270 & 0.037 & Solanum & 59 & 0.008 & Attalea & 61 & 0.008 & & & \\
\hline Nopalea & 220 & 0.030 & Sida & 56 & 0.008 & Thrinax & 57 & 0.008 & & & \\
\hline Mitrocereus & 50 & 0.007 & Physalis & 55 & 0.008 & Cocos & 6 & 0.001 & & & \\
\hline Otras (0) & 0 & 0.000 & Otras (10) & 240 & 0.033 & Otras (0) & 0 & 0.000 & Otras (0) & 0 & 0.000 \\
\hline Total (10) & 7764 & 1.062 & Total (20) & 2961 & 0.405 & Total (10) & 10252 & 1.403 & Total (5) & 832 & 0.114 \\
\hline
\end{tabular}


El género Quercus, concentró más del 27\% de los individuos. De acuerdo con Carreón-Santos \& ValdezHernández (2014) por su naturaleza en las comunidades arbóreas, los árboles tienden a ser dominantes, en virtud de que su altura les permite obtener mayor horas luz, calor, agua y nutrientes. Sin embargo, esta misma condición según Granados-Sánchez et al. (2007) hace que las comunidades arbóreas sean más vulnerables a los efectos del clima futuro.

Relación de la riqueza y diversidad de especies con los efectos del cambio climático. Al aplicar la técnica de Species richness analysis a los datos georreferenciados con distribución puntual de las 808 especies reportadas por el INFyS (CONAFOR 2014) para el Suroeste de México, se ubicaron espacialmente las regiones con el mayor número de especies (índice de riqueza de especies: IRE), de igual manera se calculó el índice de diversidad de especies dado por Shannon (IDS) que resultó con valores continuos de 0 a 3 . Estos dos indicadores se asociaron con un modelo de cambio climático (MCC) tipo A2 proyectado a 2050 mediante el algoritmo de MaxEnt (Fig. 2). El MCC resultó ser significativo con un coeficiente de ajuste (AUC) del 0.942. El AUC es una medida directa de la capacidad de discriminación del modelo, que toma valores próximos a 1 cuando existe un buen ajuste con los datos de evaluación y cercanos a 0.5 cuando el ajuste no es mejor que el obtenido por azar (Fielding \& Bell 1997).
En la Figura 2 se observa que la mayoría (> 75\%) de la cobertura territorial del hábitat climático futuro para las 808 especies analizadas, resultan ser áreas de bajo impacto (1) con una relación directa con las regiones donde coexiste el mayor número de especies y donde el índice de diversidad de especies dado por Shannon es mayor. Estos resultados difieren de lo encontrado por Garza-López et al. (2016 y 2018) para el sur de México, quienes pronostican reducciones en las coberturas de Swietenia macrophylla y Lysiloma latisiliquum del 60\% y $43 \%$ respectivamente, atribuidos a los efectos del clima futuro.

Sin embargo, los análisis de Garza-López et al. (2016 y 2018) solo consideran una especie en particular. Para nuestro caso se consideraron 808 especies de 8 formas biológicas. De acuerdo con Sakschewski et al. (2016) un ecosistema con mayor cantidad de especies puede presentar mayor estabilidad y resiliencia.

Para relacionar la resiliencia que la diversidad de especies en el Suroeste de México, presentó ante los efectos del clima futuro modelados mediante MaxEnt, se cuantificaron el número de pixeles que coincidieron de las categorías del IDS con las categorías del MCC $(-1,0,1$ y 2$)$ para ello se tomaron los valores discretos del IDS $(0,1,2$ y 3$)$ y con la técnica del vecino más próximo de SIG se obligó a que todos los pixeles del MCC coincidieran con la categoría más cercana al IDS (González de Andrés et al. 2014).

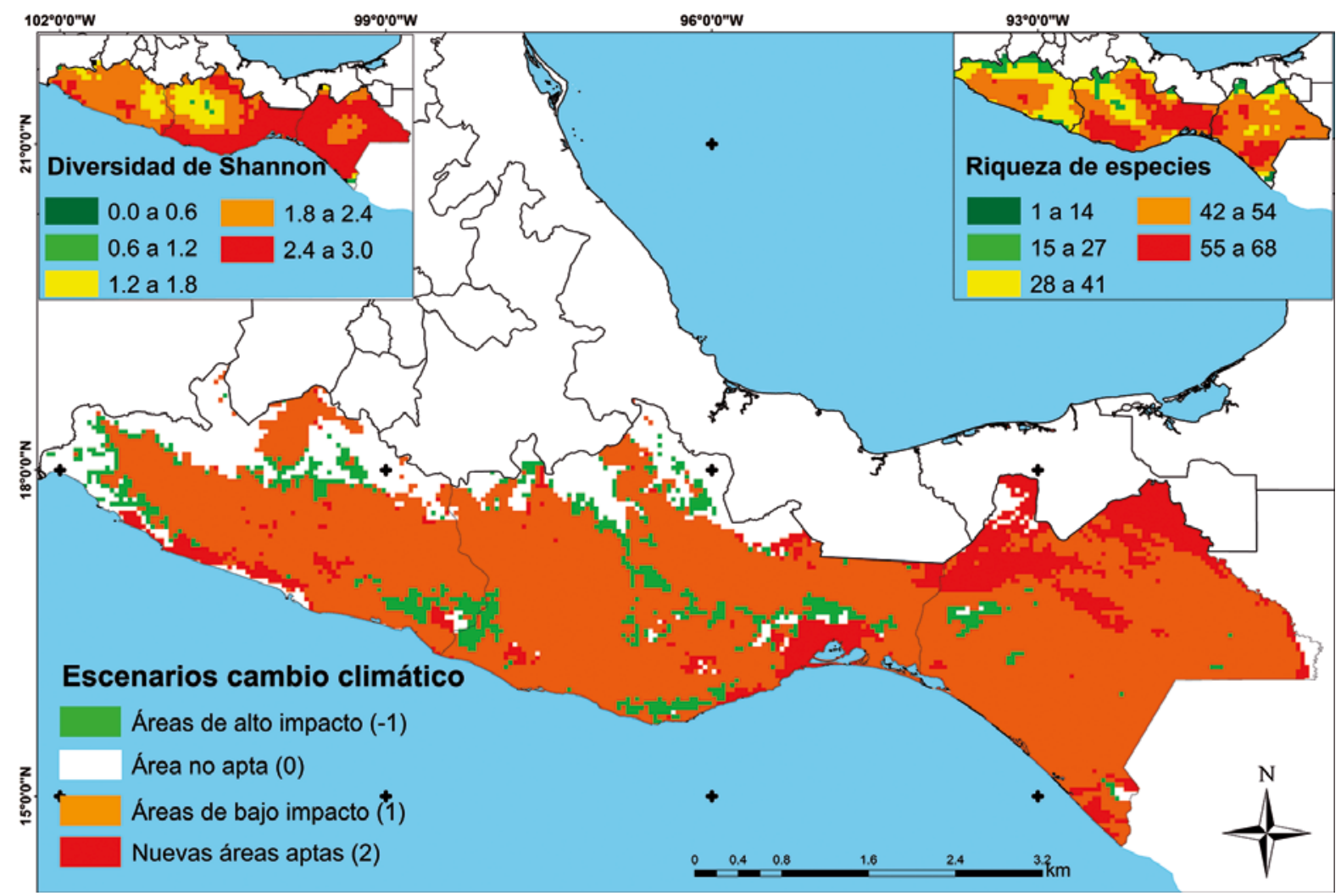

Figura 2. Representación espacial de la asociación territorial que existe entre las zonas con mayor riqueza de especies e índice de diversidad de Shannon en relación con los efectos del clima futuro proyectado a 2050 mediante un modelo de circulación global tipo A2. 
En la Figura 3 se observa que el $84 \%$ de los pixeles del MCC se concentraron en las categorías más altas del Índice de Diversidad de Shannon (2 y 3) que a su vez se asocian con los escenarios de efectos de cambio climático bajo (1: áreas de bajo impacto) y favorable (2: nuevas áreas aptas). Estos resultados coinciden con lo reportado por Gutiérrez \& Trejo (2014) quienes encontraron que para especies arbóreas de bosque templado en México, a medida que el número de especies aumenta, los efectos del cambio climático son menores; esto se explica según Chambers et al. (2019) por la diversidad ecológica.

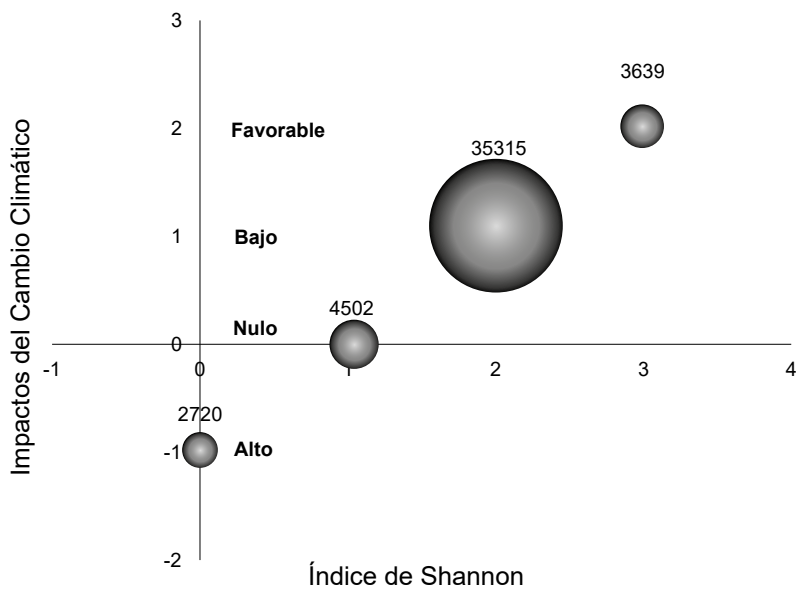

Figura 3. Relación de número de pixeles que coinciden para las categorías del modelo de cambio climático $(-1,0,1$, y 2$)$ con los valores discretos del índice de Shannon (0, 1, 2 y 3) obtenida mediante el procedimiento del vecino más cercano de SIG.

Para comprobar estadísticamente la bondad de los ajustes anteriores, se desarrolló un modelo de regresión lineal simple espacial en DivaGis mediante la instrucción analysis/regression. Se consideró como variable dependiente al MCC y como independiente al IDS. El modelo ajustado $\left(\mathrm{MCC}=0.8797+0.0103^{*} \mathrm{IDS}\right)$ resultó poco significativo con un coeficiente de determinación $\left(\mathrm{R}^{2}\right)$ de 0.0002. Sin embargo, de acuerdo con Elith et al. (2011) en la predicción de MCC lo que interesa, es el signo de la pendiente de regresión ya que determina la relación que existe entre las variables analizadas. De esta forma el modelo de regresión espacial ajustado predice que, a mayor diversidad existente en una región, los efectos del cambio climático serán menores.

Los modelos lineales, cuadráticos y exponenciales de regresión múltiple espacial, donde se evaluó a la varia- ble MCC como dependiente de las variables IDS e IRE se muestran en la Tabla 3. Se observan los parámetros de los tres modelos ajustados, así como su validación considerando valores mínimos y máximos de las variables independientes. Los resultados indican que a mayor IRE e IDS el MCC toma valores cercanos a 1 (áreas de bajo impacto) o 2 (nuevas áreas aptas). Estos resultados coinciden con Seidl et al. (2014) quienes encontraron que la resiliencia de los ecosistemas es mayor cuando en su estructura existe un mayor número de especies.

Escenarios de cambio climático por tipo de comunidad vegetal. Los resultados muestran que las áreas de alto impacto al clima futuro son mayores cuando solo se considera en el modelo una sola forma biológica (Fig. 4). De acuerdo al MCC empleado, las comunidades vegetales arbóreas y arbustivas presentan mayor resiliencia, este hecho coincide con que son las comunidades con el mayor número de especies. GómezMendoza et al. (2008) pronosticaron para la misma región de estudio que los géneros arbóreos Quercus y Pinus son los más vulnerables al cambio climático, este resultado difiere del nuestro. Sin embargo, los pronósticos de Gómez-Mendoza et al. (2008) consideran MCC por género, a diferencia del nuestro que contempló las 303 especies de comunidades arbóreas reportadas por el INFyS para el área de estudio.

Las formas biológicas de cactus, palma y xerófita presentaron la menor resiliencia a los MCC, si se consideran sus coberturas actuales en relación con las áreas de alto impacto pronosticadas. Este hecho se explica según Santiago-Vera et al. (2018) debido a que la vulnerabilidad de las especies al cambio climático aumenta si sus poblaciones son reducidas. Por su parte Gómez-Mendoza et al. (2008) considera que la vulnerabilidad de estas formas biológicas se debe a su baja tolerancia térmica, lo que explica en nuestro análisis, el por qué la estacionalidad en la temperatura (BIO4) fue la variable que más contribuyó en las predicciones de los MCC (Tabla 4).

La tolerancia térmica de las formas biológicas ha sido reportada por Fossa et al. (2004) y Hatfield \& Prueger (2015) quienes sugieren que las especies de plantas más vulnerables a la extinción y cambios en su distribución son aquéllas con bajas tolerancias a las variaciones de temperatura. Para la región de estudio Gómez-Mendoza et al. (2008) pronostican que las temperaturas se incrementaran para 2050 de 1.5 a $2.5^{\circ} \mathrm{C}$, pasando de temperaturas promedio de $23^{\circ} \mathrm{C}$ a poco más de $25^{\circ} \mathrm{C}$.

Tabla 3. Estimación y validación de modelos de regresión múltiple espacial que relacionan los efectos del cambio climático en la región Suroeste de México con la diversidad de especies presentes.

\begin{tabular}{|c|c|c|c|c|c|c|c|c|c|c|}
\hline \multicolumn{5}{|c|}{ Parametros de los modelos } & \multicolumn{6}{|c|}{ Validación de los modelos } \\
\hline \multirow{2}{*}{ Modelo } & \multirow{2}{*}{ Intercepto } & \multicolumn{2}{|c|}{ Indices } & \multirow[b]{2}{*}{$\mathbf{R}^{2}$} & \multicolumn{3}{|c|}{ Valores Minimos } & \multicolumn{3}{|c|}{ Valores Máximos } \\
\hline & & Shannon & Riqueza & & Shannon & Riqueza & Predicción & Shannon & Riqueza & Predicción \\
\hline Lineal & 0.0010 & 0.5589 & -0.0149 & 0.4846 & 0 & 1 & -0.0139 & 3 & 5 & 1.6032 \\
\hline Cuadrático & 0.0013 & -0.0971 & 0.0971 & 0.4248 & 0 & 1 & 0.0984 & 3 & 5 & 1.5549 \\
\hline Exponencial & 0.0679 & -0.0667 & 0.1057 & 0.4386 & 0 & 1 & 0.2885 & 3 & 5 & $\geq 2.0000$ \\
\hline
\end{tabular}




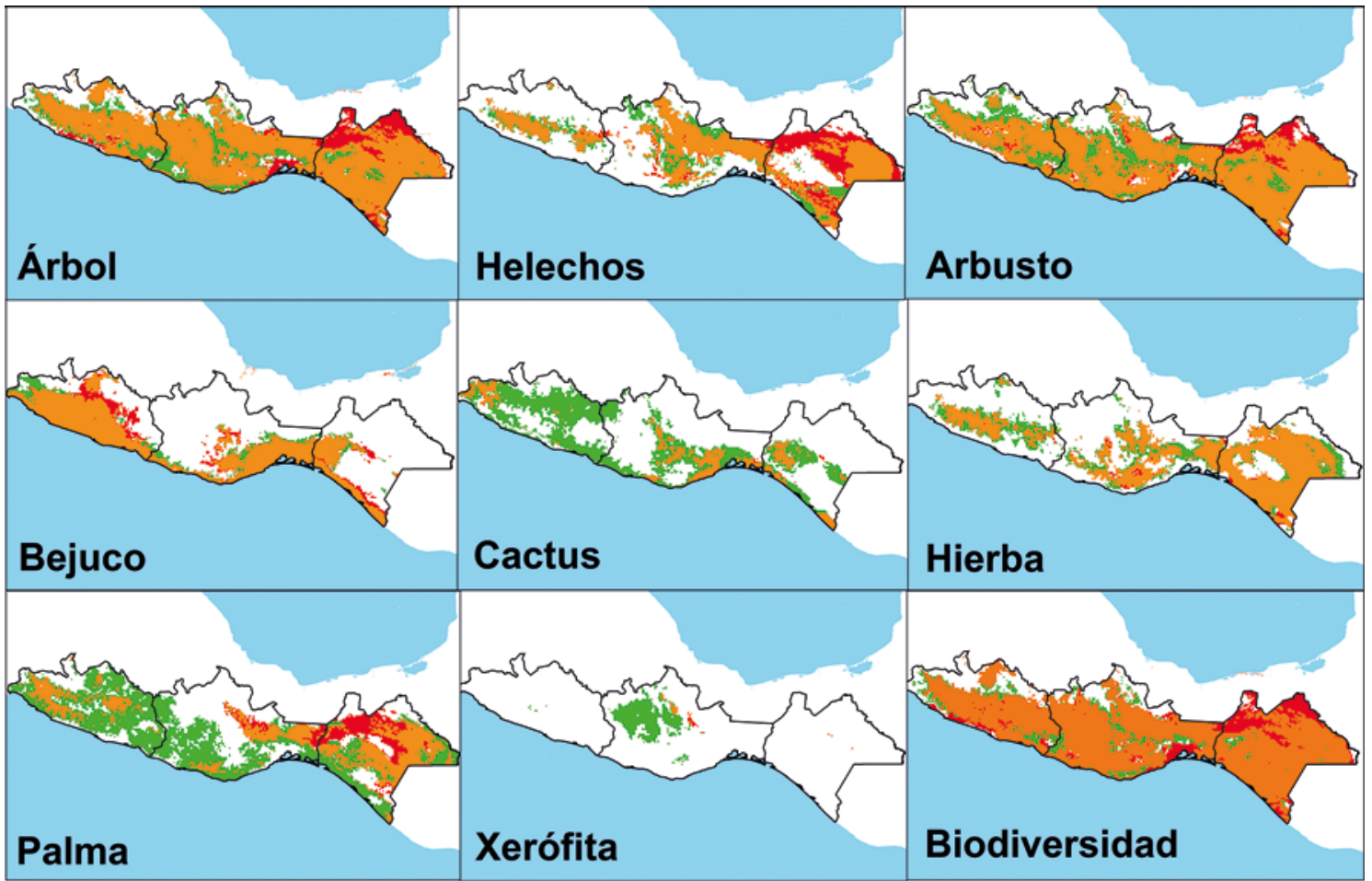

Área no apta $\quad$ Áreas de alto impacto

Áreas de bajo impacto

Nuevas áreas aptas

Figura 4. Escenarios de cambio climático de la biodiversidad y cada una de las ocho formas biológicas presentes en el Suroeste de México.

Tabla 4. Variables bioclimáticas que más contribuyen en la predicción de escenarios de clima futuro; y cobertura en $\mathrm{km}^{2}$ de los escenarios de cambio climático por biodiversidad y comunidad vegetal presentes en el Suroeste de México.

\begin{tabular}{|c|c|c|c|c|c|c|c|c|c|c|c|c|}
\hline \multirow{2}{*}{$\begin{array}{l}\text { Forma } \\
\text { biológica }\end{array}$} & \multirow{2}{*}{$\begin{array}{l}\text { Individuos } \\
\text { (n) }\end{array}$} & \multicolumn{2}{|c|}{ Área no apta } & \multicolumn{2}{|c|}{ Área de alto impacto } & \multicolumn{2}{|c|}{ Área de bajo impacto } & \multicolumn{2}{|c|}{ Nuevas areas } & \multirow{2}{*}{ AUC } & \multirow{2}{*}{ Variables* } & \multirow{2}{*}{$\begin{array}{c}\text { Contribución } \\
(\%)\end{array}$} \\
\hline & & $\mathbf{k m}^{2}$ & $\%$ & $\mathbf{k m}^{2}$ & $\%$ & $\mathbf{k m}^{2}$ & $\%$ & $\mathbf{k m}^{2}$ & $\%$ & & & \\
\hline \multirow[t]{2}{*}{ Árbol } & 660366 & 22510.91 & 9.75 & 28282.94 & 12.25 & 165080.00 & 71.5 & 15007.27 & 6.50 & 0.942 & $\mathrm{BIO4}$ & 77.50 \\
\hline & & & & & & & & & & & $\mathrm{BIO7}$ & 6.30 \\
\hline \multirow[t]{3}{*}{ Helechos } & 898 & 115648.35 & 50.09 & 21633.56 & 9.37 & 76467.83 & 33.12 & 17131.38 & 7.42 & 0.986 & $\mathrm{BlO} 4$ & 64.80 \\
\hline & & & & & & & & & & & $\mathrm{BIO13}$ & 7.40 \\
\hline & & & & & & & & & & & $\mathrm{BIO} 15$ & 7.40 \\
\hline \multirow[t]{2}{*}{ Arbustos } & 46111 & 29922.19 & 12.96 & 34793.78 & 15.07 & 152681.68 & 66.13 & 13483.46 & 5.84 & 0.955 & $\mathrm{BlO} 4$ & 76.50 \\
\hline & & & & & & & & & & & $\mathrm{BlO} 7$ & 5.40 \\
\hline \multirow[t]{4}{*}{ Bejuco } & 1773 & 144185.26 & 62.45 & 7965.4 & 3.45 & 68225.37 & 29.55 & 10505.09 & 4.55 & 0.979 & $\mathrm{BIO} 4$ & 34.00 \\
\hline & & & & & & & & & & & $\mathrm{BlO7}$ & 23.40 \\
\hline & & & & & & & & & & & $\mathrm{BIO6}$ & 19.30 \\
\hline & & & & & & & & & & & BIO19 & 15.50 \\
\hline \multirow[t]{2}{*}{ Cactus } & 7764 & 122898.02 & 53.23 & 96092.72 & 41.62 & 10966.85 & 4.75 & 923.52 & 0.40 & 0.982 & $\mathrm{BlO} 4$ & 43.80 \\
\hline & & & & & & & & & & & BIO19 & 36.00 \\
\hline \multirow[t]{2}{*}{ Hierba } & 2961 & 118003.34 & 51.11 & 30383.96 & 13.16 & 80485.16 & 34.86 & 2008.67 & 0.87 & 0.975 & $\mathrm{BlO4}$ & 75.20 \\
\hline & & & & & & & & & & & $\mathrm{BlO7}$ & 6.70 \\
\hline \multirow[t]{2}{*}{ Palma } & 10252 & 53148.83 & 23.02 & 120427.59 & 52.16 & 36456.13 & 15.79 & 20848.57 & 9.03 & 0.975 & $\mathrm{BlO} 4$ & 71.60 \\
\hline & & & & & & & & & & & BIO11 & 4.20 \\
\hline \multirow[t]{2}{*}{ Xerófita } & 832 & 202736.71 & 87.81 & 23596.05 & 10.22 & 3994.24 & 1.73 & 554.11 & 0.24 & 0.996 & $\mathrm{BIO} 10$ & 41.40 \\
\hline & & & & & & & & & & & $\mathrm{BIO} 4$ & 37.30 \\
\hline \multirow[t]{2}{*}{ Biodiversidad } & 730957 & 22510.91 & 9.75 & 13598.9 & 5.89 & 176577.88 & 76.48 & 18193.43 & 7.88 & 0.942 & $\mathrm{BIO} 4$ & 77.20 \\
\hline & & & & & & & & & & & $\mathrm{BlO7}$ & 6.70 \\
\hline
\end{tabular}

*BIO4= Estacionalidad en temperatura (desviación estándar*100). BIO6= Temperatura mínimo en el mes más frío. BIO7= Rango anual de temperatura. $\mathrm{BIO} 10=$ Temperatura promedio del trimestre más caluroso. BIO11= Temperatura promedio del trimestre más frío. BIO13= Precipitación en el mes más húmedo. $\mathrm{BIO} 15=$ Estacionalidad de la precipitación (Coeficiente de Variación). BIO19= Precipitación en el trimestre más frío. 
Estos resultados coinciden con nuestras estimaciones, donde los gradientes de temperatura son los que más aportan a las predicciones de los MCC (Tabla 4). Sin embargo, para nuestro caso, a diferencia de los trabajos de Fossa et al. (2004), Gómez-Mendoza et al. (2008) y Hatfield \& Prueger (2015), se consideró la diversidad de especies como un factor de resiliencia.

Conclusión. Los escenarios de cambio climático a 2050 para las formas biológicas que coexisten en el Suroeste de México, mostraron que las áreas de alto impacto donde una especie existe en la actualidad, pero en el futuro es probable que no, aumentó cuando se analizaron las formas biológicas por separado. El ecosistema mostró mayor resiliencia cuando se consideró toda la biodiversidad. Así mismo los modelos de regresión espacial simple y múltiple estimaron que a mayor riqueza y diversidad de especies mayor es la resiliencia. Las formas biológicas de cactus, palma y xerófita fueron las que presentaron mayor vulnerabilidad al cambio climático, las variaciones en la estacionalidad de la temperatura, resultó ser el factor que condicionará su distribución en un futuro. Sin embargo, se debe considerar que en la presente investigación se analizaron los cambios en la distribución de especies como consecuencia del cambio climático en escenario a 2050, sin ponderar la importancia de la función que las especies desempeñan en el ecosistema. Para la construcción del IRE e IDS se asumió que todas las especies son iguales en el ecosistema.

\section{Literatura citada}

Brönnimann S. 2015. Climatic Changes Since 1700. In: Climatic Changes Since 1700. Advances in Global Change Research. Springer, Cham. Pp. 167-321. https://doi. org/10.1007/978-3-319-19042-6 4

Comisión Nacional Forestal (CONAFOR). 2014. Inventario Nacional Forestal y de Suelos. <http://transparencia01. cnf.gob.mx/OpenData/Inventario/INFYS Microcuenca $20092014 />$. Acceso 12/11/2018.

Comisión Nacional Forestal (CONAFOR). 2018. Inventario Nacional Forestal y de Suelos: Informe de resultados 2009-2014. <https://n9.cl/zwm3t>. Acceso 18/02/2021.

Carreón-Santos R, Valdez-Hernández J. 2014. Estructura y diversidad arbórea de vegetación secundaria derivada de una selva mediana subperennifolia en Quintana Roo. Revista Chapingo serie ciencias forestales y del ambiente 20(1): 119-130. http://dx.doi. org/10.5154/r.rchscfa.2013.06.023

Chambers JC, Allen CR, Cushman SA. 2019. Operationalizing ecological resilience concepts for managing species and ecosystems at risk. Frontiers in Ecology and Evolution 7(241): 1-27. https://doi.org/10.3389/ fevo.2019.00241

Cuevas-Coeto A, Vera-Castillo Y, Cuevas-Sánchez J. 2019. Resiliencia y sostenibilidad de agroecosistemas tradicionales de México: Totonacapan. Revista Mexicana de Ciencias Agrícolas 10(1): 165-175. http://dx.doi. org/10.29312/remexca.v10i1.1789

DivaGis. 2019. DIVA-GIS. Version 7.5. A geographic information system for the analysis of species distribution data. $<$ http://www.diva-gis.org/>. Acceso 21/01/2019.
Dumitrescu A, Bojariu R, Birsan MV, Marin L, Manea A. 2015. Recent climatic changes in Romania from observational data (1961-2013). Theor Appl Climatol 122: 111119. https://doi.org/10.1007/s00704-014-1290-0

Dymond CC, Tedder S, Spittlehouse DL, Raymer B, Hopkins K, McCallion K, Sandland J. 2014. Diversifying managed forests to increase resilience. Canadian Journal of Forest Research 44(10): 1196-1205. https://doi. org/10.1139/cjfr-2014-0146

Elith J, Phillips SJ, Hastie T, Dudík M, Chee YE, Yates CJ. 2011. A statistical explanation of MaxEnt for ecologists. Diversity and Distributions 17(1): 43-57. https://doi. org/10.1111/j.1472-4642.2010.00725.x

Fielding AH, Bell JF. 1997. A review of methods for the assessment of prediction errors in conservation presence absence models. Environmental Conservation 24(1): 38-49. https://doi.org/10.1017/ $\underline{\text { S0376892997000088 }}$

Fossa A. 2004. Biodiversity patterns of vascular plant species in mountain vegetation in the Faroe Islands. Diversity and Distribution 10: 217-223. https://doi. org/10.1111/j.1366-9516.2004.00080.x

Fuentes-Franco R, Coppola E, Giorgi F, Pavia EG, Diro GT, Graef F. 2015. Inter-annual variability of precipitation over Southern Mexico and Central America and its relationship to sea surface temperature from a set of future projections from CMIP5 GCMs and RegCM4 CORDEX simulations. Climate Dynamics 45(1-2): 425-440. https://doi.org/10.1007/s00382-014-2258-6

Garza-López M, Ortega-Rodríguez J, Zamudio-Sánchez F, LópezToledo J, Domínguez-Álvarez F, Sáenz-Romero C. 2018. Modificación del hábitat para lysiloma latisiliquum (L.) benth (Tzalam) por el cambio climático. Revista Fitotecnia Mexicana 41(2): 127-135.

Garza-López M, Ortega-Rodríguez J, Zamudio-Sánchez F, LópezToledo J, Domínguez-Álvarez F, Sáenz-Romero C. 2016. Calakmul como refugio de Swietenia macrophylla King ante el cambio climático. Botanical Sciences 94: 43-50. http://dx.doi.org/10.17129/botsci.500

Gómez-Mendoza L, Galicia L, Aguilar-Santelises R. 2008. Sensibilidad de grupos funcionales al cambio climático en la Sierra Norte de Oaxaca, México. Investigaciones Geográficas 67: 76-100.

González de Andrés E, Traba-Díaz J, Seoane-Pinilla J, MoralesPrieto M. 2014. Aplicaciones de SIG y Teledetección en Ecología. Guión de prácticas de la asignatura. Universidad Autónoma de Madrid. <http://hdl.handle. net/10486/660536>. Acceso 11/08/2019.

Granados-Sánchez D, López-Ríos G, Hernández-García M. 2007. Ecología y silvicultura en bosques templados. Revista Chapingo serie ciencias forestales y del ambiente 13(1): 67-83.

Gutiérrez E, Trejo I. 2014. Efecto del cambio climático en la distribución potencial de cinco especies arbóreas de bosque templado en México. Revista mexicana de biodiversidad 85(1): 179-188. http://dx.doi.org/10.7550/ $\underline{\mathrm{rmb} .37737}$

Halffter G. 2017. La zona de transición mexicana y la megadiversidad de México: del marco histórico a la riqueza actual. Dugesiana 24(2): 77-89. https://doi. org/10.32870/dugesiana.v24i2.6572

Hatfield J, Prueger J. 2015. Temperature extremes: Effect on plant growth and development. Weather and climate extremes 10: 4-10. https://doi.org/10.1016/j. wace.2015.08.001 
Hijmans R, Cameron E, Parra L, Jones G, Jarvis A. 2005. Very high resolution interpolated climate surfaces for global land areas. International Journal of Climatology 25(15): 1965-1978. https://doi.org/10.1002/ joc. 1276

Hijmans RJ, Guarino L, Cruz M, Rojas E. 2001. Computer tools for spatial analysis of plant genetic resources data: 1 . DIVA-GIS. Plant genetic resources newsletter 1: 15-19.

Intergovernmental Panel on Climate Change (IPCC). 2013. Fifth assessment report, Climate change 2013: The Physical Science Basis. <http://www.ipcc.ch/report/ar5/ wg1/>. Acceso 24/05/2019.

Lindner M, Fitzgerald JB, Zimmermann NE, Reyer C, Delzon $\mathrm{S}$, van der Maaten E, Schelhaas MJ, Lasch P, Eggers J, van der Maaten-Theunissen M, Suckow F, Psomas A Poulter B, Hanewinkel M. 2014. Climate change and European forests: what do we know, what are the uncertainties, and what are the implications for forest management?. Journal of environmental management 146: 69-83. https://doi.org/10.1016/i.jenvman.2014.07.030

Lloret F. 2012. Vulnerabilidad y resiliencia de ecosistemas forestales frente a episodios extremos de sequía. Revista Ecosistemas 21(3): 85-90. https://doi.org/10.7818/ ECOS.2012.21-3.11

MaxEnt. 2019. Maxent software for modeling species niches and distributions. Version 3.4.1. <http://biodiversityinformatics.amnh.org/open source/maxent/>. Acceso 25/01/2019.

McKenney D, Pedlar J, Lawrence K, Campbell K, Hutchinson M. 2007. Potential impacts of climate change on the distribution of North American trees. BioScience 57(11): 939-948. https://doi.org/10.1641/B571106

Monterroso-Rivas A, Gómez-Díaz J, Tinoco-Rueda J. 2013. Bosque mesófilo de montaña y escenarios de cambio climático: una evaluación en Hidalgo, México. Revista Chapingo. Serie Ciencias Forestales y del Ambiente 19 (1): 29-43. https://doi.org/10.5154/r.rchscfa.2012.03.029

Moritz C, Agudo R. 2013. The future of species under climate change: resilience or decline?. Science 341(6145): 504-508. https://doi: 10.1126 / science.1237190

Phillips S, Anderson R, Schapire R. 2006. Maximum entropy modeling of species geographic distributions. Ecological Modelling 190(3-4): 231-259. https://doi. org/10.1016/i.ecolmodel.2005.03.026

Pliscoff P, Fuentes-Castillo T. 2011. Modelación de la distribución de especies y ecosistemas en el tiempo y en el espacio: una revisión de las nuevas herramientas y enfoques disponibles. Revista de Geografía Norte Grande 48(1): 61-79. http://dx.doi.org/10.4067/ S0718-34022011000100005

Sakschewski B, von Bloh W, Boit A, Poorter L, Peña-Claros M, Heinke J, Joshi J, Thonicke K. 2016. Resilience of Amazon forests emerges from plant trait diversity. Nature Climate Change 6(11): 1032-1036. https://doi. org/10.1038/nclimate3109

Santiago-Vera T, García-Millán M, Michael-Rosset P. 2018. Enfoques de la resiliencia ante el cambio climático. Agricultura, sociedad y desarrollo 15(4): 531-539.

Sarukhán J, Urquiza-Haas T, Koleff P, Carabias J, Dirzo R, Ezcurra E, Cerdeira-Estrada S, Soberón J. 2015. Strategic actions to value, conserve, and restore the natural capital of megadiversity countries: The case of Mexico. BioScience 65(2): 164-173. https://doi.org/10.1093/biosci/biu195
Scheldeman X, Willemen L, d'Eeckenbrugge GC, Romeijn-Peeters E, Restrepo MT, Romero Motoche J, Jiménez D, Lobo M, Medina CT, Reyes C, Rodríguez D, Ocampo JA, Van Damme P, Goetgebeur P. 2007. Distribution, diversity and environmental adaptation of highland papayas (Vasconcellea spp.) in tropical and subtropical America. Biodiversity and Conservation 16(6): 18671884. https://doi.org/10.1007/s10531-006-9086-X

Seidl R, Rammer W, Spies T. 2014. Disturbances legacies increase the resilience of forest ecosystem structure, composition, and functioning. Ecological Applications 24(8): 2063-2077. https://doi.org/10.1890/14-0255.1

Van Zonneveld M, Jarvis A, Dvorak W, Lema G, Leibing C. 2009a. Climate change impact predictions on Pinus patula and Pinus tecunumanii populations in Mexico and Central America. Forest Ecology and Management 257(7): 1566-1576. https://doi.org/10.1016/i.fore$\underline{\text { co.2008.12.027 }}$

Van Zonneveld M, Koskela J, Vinceti B, Jarvis A. 2009b. Impact of climate change on the distribution of tropical pines in Southeast Asia. Unasylva 231(232): 24-29.

Whitney CK, Bennett NJ, Ban NC, Allison EH, Armitage D, Blythe JL, Burt JM, Cheung W, Finkbeiner EM, Kaplan-Hallam M, Perry I, Turner NJ, Yumagulova L. 2017. Adaptive capacity: from assessment to action in coastal socialecological systems. Ecology and Society 22(2):1-30. https://doi.org/10.5751/ES-09325-220222

\section{Agradecimientos / Acknowledgments:}

Este trabajo forma parte de la Catedra-Conacyt 364 titulada: Reconversión productiva sustentable para el desarrollo de los productores rurales de Campeche. Se agradece a las autoridades del Instituto Tecnológico Superior de Venustiano Carranza por las facilidades brindadas para la defensa de tesis de la Licenciatura en Ingeniería Forestal del segundo autor.

\section{Conflicto de intereses / Competing interests:}

Los autores no incurren en conflictos de intereses.

\section{Rol de los autores / Authors Roles:}

ASF: conceptualización y diseño del estudio, análisis estadísticos y redacción del manuscrito final. IIVC: elaboración de mapas mediante SIG y análisis de la información. LMPR: redacción del manuscrito original. ECA: análisis de la información y revisión de manuscrito original. FAC: revisión de datos y revisión del manuscrito final. JBO: revisión, seguimiento de resultados, redacción del manuscrito final.

\section{Fuentes de financiamiento / Funding:}

Esta investigación no recibió ninguna subvención específica de ninguna agencia de financiación, ni del sector comercial o sin fines de lucro.

\section{Aspectos éticos / legales; Ethics / legals:}

Los autores declaran que no violaron ni omitieron normas éticas o legales en esta investigación. 
Página en banco

Blank page 